\title{
Impact of 'ADL self-management module' on health related quality of life among road traffic accidents' patients: a quasi-experimental research approach
}

\begin{abstract}
Background: Road traffic accidents represent a community and governmental concern in Saudi Arabia and worldwide. In addition, road traffic accidents injuries are among the main causes of physical impairment and functional dependency. Therefore, performance of activity of daily living of those injured patients are severely affected due to loss of independence. Subsequently, quality of life is affected as well. Objective: To develop, implement and evaluate the impact of "ADL self-management module" on health related quality of life among road traffic accidents' patients. Methods/design: A quasi experimental study design will be conducted in Saudi Arabia in which 152 orthopedic patients with lower extremities fractures due to road traffic accidents who have difficulty in carrying out daily activity will be recruited from the study hospitals based on Barthel Index. The patients in the intervention group will provided with the ADL Self-Management Module in a form of an educational material. They will be supplemented in that module with knowledge, skills and confidence to engage in self-management of activity of daily living tasks independently, whereas the control group will receive the usual care. Descriptive analysis, chi square and Generalized Estimation Equation, Independent $t$ test will be used to test the differences of activity of daily living and health related quality of life between and within the groups. Discussion: To our knowledge, this is the first experimental study that will conducted in Saudi Arabia on ADL self-management to improve the quality of life of Road Traffic Accident survivors who have difficulty in performing daily activities.
\end{abstract}

Keyword: Road traffic accidents; Self- management; Activity of daily living; Health related quality of life; Quasi rxperiment 